\title{
Inhaltsübersicht
}

\section{Band 4}

\section{BESONDERER TEIL}

Erster Abschnitt. Friedensverrat, Hochverra: und Gefährdung des demokratischen Rechtsstaates

$\begin{array}{lcc}\text { Erster Titel. Friedensverrat } & \text { Erschienen } & \text { Bearbeiter } \\ \text { Vorbemerkungen zu den } \S \S 80 \mathrm{ff} & 7 / 1980 & \text { Willms } \\ \S \S 80,80 \mathrm{a} & 7 / 1980 & \text { Willms }\end{array}$

Zweiter Titel. Hochverrat
$\S \S 81-83 \mathrm{a}$
$7 / 1980$
Willms

Dritter Titel. Gefährdung des demokratischen Rechtsstaates $\S \S 84-91$

$7 / 1980$

Willms

Vierter Titel. Gemeinsame Vorschriften $\S \S 92-92 b$

7/1980 Willms

Zweiter Abschnitt. Landesverrat und Gefährdung der äußeren Sicherheit

Vorbemerkungen zu den $\$ \S 93$ ff $\S \S 93-101$ a
5/1987

$5 / 1987$
Träger

Träger

Dritter Abschnitt. Straftaten gegen ausländische Staaten

Vorbemerkungen zu den $\S \S 102$ ff $\S \S 102-104 \mathrm{a}$

4/1981

Willms

4/1981 Willms

Vierter Abschnitt. Straftaten gegen Verfassungsorgane sowie bei Wahlen und Abstimmungen

Vorbemerkungen zu den $\S \S 105 \mathrm{ff}$ $\S \S 105-108 \mathrm{~d}$
4/1981

4/1981
Willms

Willms 
Fünfter Abschnitt. Straftaten gegen die Landesverteidigung

Vorbemerkungen zu den $\S \S 109$ ff $\S 109-109 \mathrm{k}$

$4 / 1981$

$4 / 1981$

Schroeder

Schroeder

Sechster Abschnitt. Widerstand gegen die Staatsgewalt

Vorbemerkungen zu den $\S \S 110 \mathrm{ff}$ $\S \S 111-122$

$9 / 1978$

$9 / 1978$

von Bubnoff

von Bubnoff

Siebenter Abschnitt. Straftaten gegen die öffentliche Ordnung

\begin{tabular}{|c|c|c|}
\hline Vorbemerkungen zu den $\S \S 123$ ff & $12 / 1978$ & von Bubnoff \\
\hline$\S \S 123,124$ & $12 / 1978$ & Schäfer \\
\hline Vorbemerkungen zu den $\S \S 125 \mathrm{ff}$ & $10 / 1978$ & von Bubnoff \\
\hline$\S \S 125-131$ & $10 / 1978$ & von Bubnoff \\
\hline$\S 132$ & $10 / 1978$ & Herdegen \\
\hline$\S \S 132 a-137$ & $10 / 1978$ & von Bubnoff \\
\hline$\S \S 138-141$ & $10 / 1978$ & Hanack \\
\hline $\begin{array}{l}\S 142 \\
\text { (§ } 143 \text { weggefallen) }\end{array}$ & $12 / 1978$ & Rüth \\
\hline$\$ \S 144,145$ & $12 / 1978$ & Herdegen \\
\hline $\begin{array}{l}\S 145 \mathrm{a} \\
(\S 145 \mathrm{~b} \text { weggefallen) }\end{array}$ & $12 / 1978$ & Hanack \\
\hline$\$ 145 \mathrm{c}$ & $12 / 1978$ & Horstkotte \\
\hline$\S 145 d$ & $12 / 1978$ & Willms \\
\hline
\end{tabular}

Achter Abschnitt. Geld- und Wertzeichenfälschung

Vorbemerkungen zu den $\S \S 146 \mathrm{ff}$

$12 / 1978$

$12 / 1978$

Herdegen

Herdegen

$\S \S 146-152$

$12 / 1978$

Neunter Abschnitt. Falsche uneidliche Aussage und Meineid

Vorbemerkungen zu den $\S \S 153$ ff

$12 / 1978$

12/1978 Willms

Willms

$\S \S 153-163$

Zehnter Abschnitt. Falsche Verdächtigung

$\S \S 164,165$

$12 / 1978$

Herdegen

(VI) 
Elfter Abschnitt. Straftaten, welche sich auf Religion und Weltanschauung beziehen

Vorbemerkungen zu den $\S \S 166 \mathrm{ff}$ $\S \S 166-168$

$12 / 1984$

$12 / 1984$

Dippel

Dippel

Zwölfter Abschnitt. Straftaten gegen den Personenstand, die Ehe und die Familie

Vorbemerkungen zu den $\S 169 \mathrm{ff}$ $\S \S 169-173$

$\begin{array}{ll}5 / 1986 & \text { Dippel } \\ 5 / 1986 & \text { Dippel }\end{array}$

Dreizehnter Abschnitt. Straftaten gegen die sexuelle Selbstbestimmung

Vorbemerkungen zu den $\$ \$ 174 \mathrm{ff}$ $\S \S 174-184 \mathrm{c}$

$12 / 1984$

Laufhütte

12/1984 Laufhütte 


\section{Band 5}

$\begin{array}{lcc} & \text { Erschienen } & \text { Bearbeiter } \\ & \text { Vierzehnter Abschnitt. Beleidigung } & \\ \text { Vorbemerkungen zu den } \S \S 185 \mathrm{ff} & 12 / 1988 & \text { Herdegen } \\ \S \S 185-200 & 12 / 1988 & \text { Herdegen }\end{array}$

Fünfzehnter Abschnitt. Verletzung des persönlichen Lebens und Geheimbereichs

Vorbemerkungen zu den $\S \S 201 \mathrm{ff}$

$\begin{array}{ll}8 / 1988 & \text { Träger } \\ 8 / 1988 & \text { Träger } \\ 8 / 1988 & \text { Jähnke } \\ 8 / 1988 & \text { Träger }\end{array}$

Sechzehnter Abschnitt. Straftaten gegen das Leben

Vorbemerkungen zu den $\S \S 211 \mathrm{ff}$

$2 / 1980$

Jähnke

$\S \S 211-217$

2/1980 Jähnke

Vorbemerkungen zu den $\S \S 218$ ff

$10 / 1983$

$\S \S 218-220 \mathrm{a}$

$10 / 1983$

$\S \S 221,222$

4/1981

Jähnke

Jähnke

Jähnke

Siebzehnter Abschnitt. Körperverletzung
Vorbemerkungen zu den $\S \S 223$ ff
4/1981
H.-J. Hirsch
$\S \S 223-233$
$4 / 1981$
H.-J. Hirsch

Achtzehnter Abschnitt. Straftaten gegen die persönliche Freiheit

Vorbemerkungen zu den $\S \S 234 \mathrm{ff}$

$\begin{array}{ll}8 / 1979 & \text { Vogler } \\ 8 / 1979 & \text { Vogler } \\ 8 / 1986 & \text { Schäfer }\end{array}$

$\S \S 234-238$

$8 / 1986$

Schäfer

Neunzehnter Abschnitt. Diebstahl und Unterschlagung

Vorbemerkungen zu den $\S \S 242$ ff

$8 / 1988$

Ruß

$\S \S 242-248 \mathrm{c}$

$8 / 1988$

$\mathrm{RuB}$

(VIII) 
Zwanzigster Abschnitt. Raub und Erpressung

$\S \S 249-252$

$\S \S 253-256$
$12 / 1982$

$12 / 1982$
Herdegen

Lackner

Einundzwanzigster Abschnitt. Begünstigung und Hehlerei

Vorbemerkungen zu den $\S 257 \mathrm{ff}$

$\S \S 257-262$
$12 / 1982$

$12 / 1982$
RuB

Ruß 
Band 6

Erschienen Bearbeiter

Zweiundzwanzigster Abschnitt. Betrug und Untreue

$\S 263$

$\S 264$

$\S \S 265,265 \mathrm{a}$

$\S 265 \mathrm{~b}$

$\S \S 266$
$9 / 1979$

$9 / 1979$

$9 / 1979$

$9 / 1979$

$9 / 1979$
Lackner Tiedemann Lackner Tiedemann Hübner

Dreiundzwanzigster Abschnitt. Urkundenfälschung

Vorbemerkungen zu den $\S 267 \mathrm{ff}$ $\S \S 267-282$
$9 / 1982$
$9 / 1982$
Tröndle
Tröndle

Vierundzwanzigster Abschnitt. Konkursstraftaten

Vorbemerkungen zu den $\S \S 283 \mathrm{ff}$ $\S \S 283-283 \mathrm{~d}$
$12 / 1984$ $12 / 1984$
Tiedemann Tiedemann

Fünfundzwanzigster Abschnitt. Strafbarer Eigennutz

Vorbemerkungen zu den $\S \S 284 \mathrm{ff}$ $\S \S 284-287$

$\S \S 288-302 \mathrm{a}$
$11 / 1979$

$11 / 1979$

$11 / 1979$ von Bubnoff von Bubnoff Schäfer 


\section{Band 7}

Erschienen

Bearbeiter

Sechsundzwanzigster Abschnitt. Sachbeschädigung

$\S \S 303-305$

$10 / 1978$

Wolff

Siebenundzwanzigster Abschnitt. Gemeingefährliche Straftaten

$\S \S 306-311 \mathrm{c}$

$\S \S 311 \mathrm{~d}, 311 \mathrm{e}$

$\S \S 312-314$

$\$ \$ 315,316$

$\S 316 \mathrm{a}$

$\S \S 316 b, 316 \mathrm{c}$

$\S 317$

$\S \S 318-323$

$\S \S 323 \mathrm{a}, 323 \mathrm{~b}$

$\S 323 \mathrm{c}$
$10 / 1978$

$10 / 1985$

$10 / 1978$

$10 / 1978$

$10 / 1978$

$10 / 1978$

$10 / 1978$

$6 / 1985$

$6 / 1985$

$8 / 1988$
Wolff

Steindorf

Wolff

Rüth

Schäfer

Rüth

Wolff

Wolff

Spendel

Spendel

Achtundzwanzigster Abschnitt. Straftaten gegen die Umwelt

Vorbemerkungen zu den $\S \S 324 \mathrm{ff}$ $\S \S 324-330 \mathrm{~d}$
$10 / 1985$

$10 / 1985$
Steindorf

Steindorf

Neunundzwanzigster Abschnitt. Straftaten im Amte

Vorbemerkungen zu den $\S \S 331 \mathrm{ff}$

$\S \S 331-335 \mathrm{a}$

$\S 336$

( $\S 337-339$ weggefallen)

$\S 340$

( $\S 341,342$ weggefallen)

$\S \S 343-345$

( $\S 346,347$ weggefallen)

$\S 348$

( $\S 349-351$ weggefallen)

$\S \S 352-353 \mathrm{~d}$

$\S \S 354,355$

$\S 356$

$\S 357$

$\S 358$ $\begin{array}{ll}5 / 1982 & \text { Jescheck } \\ 5 / 1982 & \text { Jescheck } \\ 5 / 1982 & \text { Spendel }\end{array}$

5/1982 H.-J. Hirsch

5/1982 Jescheck

5/1982 Tröndle

5/1982 Träger

$5 / 1982 \quad$ Schäfer

$7 / 1980$ Hübner

$5 / 1982 \quad$ Jescheck

5/1982 Schäfer 
